\title{
II. ADMINISTRATION ET FINANCES DE L'UNION ASTRONOMIQUE INTERNATIONALE
}

\section{A. ADMINISTRATION DE L'UNION ASTRONOMIQUE INTERNATIONALE}

L'administration de l'UAI a pour but essentiel de lui permettre d'atteindre les objectifs qui lui sont fixés par les Statuts, c'est-à-dire faciliter les relations entre astronomes des différents pays lorsque la coopération internationale est utile ou nécessaire, et promouvoir l'étude et le développement de l'astronomie sous tous ses aspects.

En raison de la limitation de ses fonds et de son secrétariat, l'UAI dans ses débuts a surtout veillé à la réalisation du premier de ses buts. Ultérieurement, et surtout depuis la guerre, l'UAI a progressivement travaillé au second, la promotion de l'étude de l'astronomie dans tous ses domaines, et ce principalement grâce à l'aide de l'UNESCO qui a permis l'organisation d'un plus grand nombre de symposiums.

L'organisation de la coopération internationale reste toutefois l'objet principal de l'UAI; c'est une tâche que seule l'Union, libre de toute influence politique ou gouvernementale, peut remplir de façon adéquate.

Ce n'est pas seulement les résultats spectaculaires et pratiques de l'organisation internationale (l'UAI en a maint à son actif) qui indiquent dans quelle mesure les buts ont été atteints. Il ne faut pas oublier que l'UAI joue aussi le rôle symbolique, largement et librement utilisé par tous les astronomes, à titre individuel ou collectif, de l'organisation responsable sous l'égide de laquelle la coopération peut être poursuivie. Le caractère représentatif de l'Union, son niveau scientifique, sont une garantie excellente, vis-à-vis des gouvernements et des autres autorités responsables, de la valeur de ses entreprises et de l'impartialité absolue de leur organisation.

L'Union est administrée par le Président, le Secrétaire Général, le Secrétaire Général Adjoint, sous contrôle du Comité Exécutif, ainsi qu'il est précisé dans les Statuts, p. ${ }_{3} 8$ de ce volume. La composition actuelle du Comité Exécutif de l'Union Astronomique Internationale est rappelée p. 137 .

Dans sa tâche, le Secrétaire Général est assisté par un Secrétaire Adjoint, M. A. Jappel, et par du personnel de secrétariat; les affaires générales de l'UAI sont traitées actuellement à Nice (Observatoire de Nice, le Mont-Gros, o6-Nice, France), cependant que tous les problemes relatifs à l'édition des publications de l'UAI sont traités à Meudon (Observatoire de Meudon, 5 place Janssen, 92-Meudon, France). Cette extension du Secrétariat est récente. C'est à partir de 195I qu'un Secrétaire Général Adjoint était adjoint au Secrétaire Général, pendant l'année qui précédait l'expiration de son mandat, puis, depuis 1961, pendant les trois années précédant cette expiration; c'est en 1964 que le Secrétaire Général Adjoint devient ipso facto membre du Comité Exécutif. Le poste de Secrétaire Adjointe est officiellement créé en 1959. Après avoir assuré le secrétariat du Professeur Oosterhoff depuis 1953, Miss N. Splinter fut la première Secrétaire Adjointe de l'UAI, de 1959 à 1962. Miss D. A. Bell lui succéda de 1962 à 1966 . Le nombre de personnes émargeant au budget de l'UAI est actuellement de deux, mais on ne peut maintenir ce nombre à un niveau si bas que grâce à l'aide qui a toujours été apportée par les observatoires qui ont successivement accueilli le secrétariat de l'UAI.

On trouvera au début des volumes successifs des Transactions de l'UAI et depuis le volume XI, au début du tome B, le rapport d'activité du Comité Exécutif. Sa lecture, vivement recom- 
mandée à tous les Membres de l'Union, rappelle les principales entreprises menées avec l'aide de l'UAI et explique le fonctionnement administratif de l'Union.

\section{B. FINANCES DE L'UNION ASTRONOMIQUE INTERNATIONALE}

Conformément aux règles statutaires, les finances de l'UAI sont alimentées essentiellement par deux sources:

(a) les contributions des pays adhérents qui sont actuellement égales à I 56 unités par an, depuis la modification des Statuts intervenue en I 964 ; une unité valant actuellement 600 francs or (soit 195.96 U.S. \$).

(b) la subvention annuelle de l'UNESCO (attribuée par l'intermédiaire de l'ICSU) qui atteint actuellement une valeur moyenne d'environ 14000 U.S. \$).

A ces sources régulières de revenu, il faut ajouter évidemment la vente des publications, et diverses autres opérations; mais elles compensent seulement en partie les dépenses faites, dont l'impression des publications est l'une des plus lourdes.

Les dépenses de nature strictement administrative sont inférieures à $15 \%$ des revenus. Elles restent à un niveau très bas, à la fois en raison d'une administration traditionnellement orientée vers l'économie, et grâce à l'hospitalité généreuse des pays où le secrétariat a été installé. Cependant l'augmentation régulière du nombre des Membres de l'Union et de ses activités impliquera nécessairement une augmentation progressive des dépenses administratives.

La plupart des dépenses de l'Union sont consacrées à l'organisation (frais de voyages) de réunions scientifiques, au financement d'opérations particulières (propres à chacune des Commissions de l'Union), à l'impression des publications de l'UAI, et à des subventions destinées à favoriser l'échange des astronomes entre les différents pays (particulièrement dans le cas des jeunes astronomes et des pays en voie de développement).

\section{LES COMMISSIONS DE L'UNION ASTRONOMIQUE INTERNATIONALE}

L'UAI comporte actuellement $3^{8}$ Commissions dont quatre, d'un caractère administratif plutôt que scientifique, sont considérées comme des Comités du Comité Exécutif. Les autres Commissions couvrent l'ensemble des branches de l'Astronomie, y compris les aspects astronomiques de la recherche spatiale. Un certain nombre de Commissions sont concernées principalement par des techniques, mais la plupart sont définies par leurs sujets de recherche, comme le Soleil, les Planètes, la Galaxie.

La plupart des Commissions représentent la continuation du travail commencé avant la fondation de l'Union (voir ci-dessus p. 3). Beaucoup d'entre elles ont été créées en 1922 et ont depuis continué leur activité sans changement, mais un grand nombre sont entièrement nouvelles et, continuellement, de nouvelles Commissions sont créées ou modifiées; parmi les modifications les plus récentes citons la transformation de la Commission $\mathbf{1 7}$, antérieurement Commission de la Figure et du Mouvement de la Lune, en Commission de la Lune, et la création des Commissions 45 et 46 , respectivement Commission des Classifications Spectrales et Indices de Couleurs à Plusieurs Bandes et Commission de l'Enseignement de l'Astronomie.

Les Commissions ont une très grande autonomie. Chaque Commission est administrée par un Président assisté d'un Vice-Président et d'un Comité d'Organisation. L'une des tâches essentielles des Commissions est de soumettre un rapport à l'Assemblée Générale tous les trois ans. Ces rapports qui sont distribués aux Membres comme "Draft Reports", antérieurement à l'Assemblée Générale, sont un des aspects très particuliers de notre Union: ils forment actuellement une revue d'ensemble qui fait autorité sur les progrès de l'Astronomie accomplis dans toutes ses branches depuis l'Assemblée Générale précédente.

Bien entendu les fonctions et les activités des Commissions diffèrent très largement d'une 
Commission à l'autre. Un certain nombre d'entre elles limitent leurs activités à la centralisation et à l'échange d'information, ainsi qu'à l'organisation de colloques et de symposiums. Un certain nombre sont au contraire presque uniquement concernées par des programmes de coopération de recherche ou d'observation, et par des problèmes de standardisation. Mais la plupart des Commissions sont intéressées de façon plus ou moins intense par chacune de ces deux formes d'activité. L'une des tâches les plus difficiles du Comité des Finances est de donner son avis à l'Assemblée Générale au sujet de la distribution des fonds qui sont à sa disposition, entre les différents projets particuliers proposés par les différentes Commissions.

Les Commissions se réunissent principalement à l'occasion des Assemblées Générales, mais, de plus en plus, un certain nombre de Commissions continuent une activité régulière entre les Assemblées, le plus souvent par voie de correspondance, mais parfois en organisant des réunions propres à ces Commissions.

De nombreuses Commissions de l'Union ont leur propre système de circulaires (étoiles doubles, étoiles variables, etc.) et procèdent à la publication de nombreux documents de travail. 